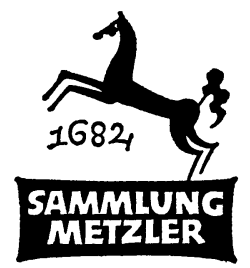

REALIEN ZUR LITERATUR ABT. D:

LITERATURGESCHICHTE 
WOLFGANG BENDER

\section{Johann Jakob Bodmer und}

Johann Jakob Breitinger

MCMLXXIII

J. B. METZLERSCHE VERLAGSBUCHHANDLUNG STUT'TGART 
ISBN 978-3-476-10113-6

ISBN 978-3-476-03833-3 (eBook)

DOI $10.1007 / 978-3-476-03833-3$

\section{II3}

(C) Springer-Verlag GmbH Deutschland 1973

Ursprünglich erschienen bei J. B. Metzlersche Verlagsbuchhandlung und Carl Ernst Poeschel Verlag GmbH in Stuttgart 1973 


\section{INHALT}

I. Materialien . . . . . . . . . . . . . . . I I

I. Der Nachlaß . . . . . . . . . . . . I I

2. Ausgaben .............. . 2

3. Bibliographien und die Bodmer-Denkschrift . . 6

4. Briefe . . . . . . . . . . . . . . . 8

II. Biographie und Persönlichkeit . . . . . . . . I4

I. Johann Jakob Bodmer . . . . . . . . . . . I4

2. Johann Jakob Breitinger . . . . . . . . 17

III. DAS Werk . . . . . . . . . . . . . . . 22

I. Die Gesellschaftsschriften . . . . . . . 22

a) Gesellschaftliche Voraussetzungen . . . . . . 22

b) \Die Discourse der Mahlern', 'Crito', Plan eines >Phantasten `............ 24

c) Wirkungen . . . . . . . . . . . . . . 27

d) $>$ Der Mahler der Sitten $\quad . \quad . \quad . \quad . \quad . \quad . \quad 28$

2. Historische Studien . . . . . . . . 30

3. Altdeutsche Studien und Textausgaben . . . . 34

4. Ubersetzung: Theorie und Praxis . . . . . . 43

s. Editionen neuerer deutscher Texte. . . . . . sI

6. Bodmers dichterische Versuche ....... ss

a) Dramen . . . . . . . . . . . . . ss

b) Biblische und Historische Epen. . . . . 58

c) Sonstige Dichtungen . . . . . . . . 6I

7. Kritisch-Ästhetische Schriften . . . . . . . 69

a) Kleinere Schriften . . . . . . . . . . . 70

b) Der /Brief-Wechsel von der Natur des Poetischen Geschmackesı. . . . . . . . . . . 79

c) Die vier Hauptschriften von $1740 / 41$. . . 85 Bodmers >Critische Abhandlung von dem Wunderbarenı ........... 85 
Breitingers >Critische Abhandlungs und Bodmers >Critische Betrachtungenı . . . . . . 87 Breitingers $>$ Critische Dichtkunst . . . . . 90

d) Wirkungen . . . . . . . . . . . . . 94

IV. Aufgaben der Forschung . . . . . . . . . I IO

REGISTER . . . . . . . . . . . . . . II4 


\section{VORBEMERKUNG}

Die Maxime der Gedankenfreiheit beherrschte das Wirken der beiden Männer, denen die Schreiber von literarhistorischen Büchern und Abhandlungen einen bescheidenen Platz als Künder neuer ästhetischer Einsichten angewiesen haben. Hätte man ihrem leidenschaftlichen Engagement für die Anerkennung und Erhaltung republikanischer Tugenden von vornherein die gebührende Aufmerksamkeit geschenkt, so wäre es kaum zu Klischeebildungen gekommen, wie wir sie in der Literaturgeschichtsschreibung häufig vorfinden. Maßgeblich für die Beurteilung ihres Werkes war der ästhetische Kanon der klassisch-idealistischen Dichtungsauffassung. An diesem gemessen, kam ihnen nichts weiter als eine Art, Vorläuferrolle $\mathrm{zu}^{\mathrm{z}}$. Daß ihr kritisch-ästhetisches Werk als gewichtiger Beitrag in dem Entwicklungsprozeß gewertet werden muß, der schließlich hinführt zur Symbolkunst im Sinne Goethes, wird kaum von der Hand zu weisen sein. Nur hat die Überbetonung dieses Aspekts zu Verzerrungen des Bodmer- und Breitinger-Bildes geführt, die literarhistorisch durch nichts zu rechtfertigen sind.

Dem kritisch-ästhetischen Bereich ihres Schaffens gilt ein Teil der vorliegenden Arbeit - aber auch eben nur ein Teil. Es geht uns nicht um die ,Rettung' eines hochgebildeten Zürcher Antisten, des Verfassers einer "Critischen Dichtkunst", oder um die des "Vaters der Jünglinge", dessen Produktion im Alter in der Tat beängstigende Formen annahm, sondern um literarhistorische Korrekturen. Daneben breiten wir Fakten aus, so wie es der Zielsetzung der ,Sammlung Metzler' entspricht. Den „Altgermanisten“, Historikern, Übersetzern und Herausgebern Bodmer und Breitinger gelten vier Kapitel der Studie, dem „Dichter" Bodmer ein weiteres. Darüber ist unseres Wissens noch nicht im größeren Zusammenhang gehandelt worden. Wir teilen die Ansicht Walther Goses (>Jahrb. d. Dt. Schillerges.( 13, 1969), daß eine Darstellung der Theologie Breitingers ein höchst wünschenswertes Unternehmen sei. Diese Arbeit kann freilich im Rahmen dieses Realienbandes nicht geleistet werden. Daß eine solche Darstellung nur das Resultat der Kooperation eines Literarhistorikers und eines Theologen sein könnte, versteht sich. 
Anregend in jeder Hinsicht waren für den Verfasser die Studien Herbert Schöfflers (»Deutscher Geist im I 8. Jahrhundert", 1956) und der meisterhafte Essay, den Martin Bodmer seinem Landsmann und Namensvetter 1943 in der Zeitschrift >Corona widmete.

Herzlich danken wir den Bibliothekaren der Zentralbibliothek Zürich und der Schweizerischen Landesbibliothek Bern für nie ermüdende Hilfsbereitschaft. Dank sei jenen Kollegen, die uns immer wieder zur Überprüfung der eigenen Ansichten veranlaßten.

Bad Münstereifel/Münster, Westf. im Spätherbst 1972

WOLFGANg Bender 


\section{Abkürzungen}

AA Wielands Gesammelte Schriften. Hrsg. von der Deutschen Kommission der Kgl. Preuß. Akademie derWissenschaften (später: der Preuß. Akad.

ADB

bearb.

Bibl.

Bibl. ält. Schriftw. Bibliothek älterer Schriftwerke der deutschen Schweiz

$\mathrm{Bl}(\mathrm{l})$.

BLVS

BPZ

BW

BWK

CNRG

DA

Denkschrift

Diss.

DLD I8. Jahrh.

DN

DNL

DVjs.

EK

FN

GDLS

gedr.

GGA

GLL

GRM

Allgemeine Deutsche Biographie

bearbeitet

Bibliothek

Blatt bzw. Blätter

Bibliothek des Litterarischen Vereins in Stuttgart

Berlinische Privilegierte Zeitung

Bodmer: Brief-Wechsel von der Natur des poetischen Geschmackes ( $173^{6}$ )

Bibliothek der schönen Wissenschaften und der freyen Künste. 1757-1765.

Critische Nachrichten aus dem Reiche der Gelehrsamkeit. 1750 und 1752.

Dissertation Abstracts

Johann Jakob Bodmer. Denkschrift zum CC.

Geburtstag. Veranlaßt vom Lesezirkel Hottingen und hrsg. von der Stiftung Schnyder von Wartensee. 1900 .

Dissertation

Deutsche Litteraturdenkmale des 18. Jahrhunderts

Deutsche Neudrucke

Deutsche National Litteratur

Deutsche Vierteljahrsschrift

Bodmer-Breitinger: Von dem Einfluß und Gebrauche der Einbildungs-Krafft. 1727 .

Freymüthige Nachrichten von neuen Büchern und andern zur Gelehrtheit gehörigen Sachen. I 744-I 75 I.

Jakob Baechtold: Geschichte der deutschen Literatur in der Schweiz. 1892.

gedruckt

Göttingische Gelehrte Anzeigen

German Life and Letters

Germanisch-Romanische Monatsschrift derWissenschaften). Berlin I $909 \mathrm{ff}$. 

Hrsg. von Heinrich Türler, Marcel Godet und Victor Attinger. 1921-1934.

Holzmann-Bohatta Michael Holzmann und Hanns Bohatta: Deutsches Anonymen-Lexikon. 1902-1928.

Hs. Handschrift

JDSG Jahrbuch der Deutschen Schillergesellschaft

Jahrh. Jahrhundert

JFDH Jahrbuch des Freien Deutschen Hochstifts

Jöcher (Forts.) Fortsetzung und Ergänzungen zu Christian Gottlieb Jöchers allgemeinem Gelehrten-Lexikon ... von Joh. Christoph Adelung. $1784 \mathrm{ff}$.

Körte Briefe der Schweizer Bodmer, Sulzer, Geßner. Aus Gleims litterarischem Nachlasse, hrsg. von Wilhelm Körte. I 804 .

LD Literarische Denkmale von verschiedenen Verfassern. 1779 .

Lfrg. $\quad$ Lieferung

LM Gotthold Ephraim Lessings sämtliche Schriften, hrsg. von Karl Lachmann, 3. auf's neue durchgesehene u. vermehrte Aufl., besorgt durch Franz Muncker. I $886 \mathrm{ff}$.

LP Litterarische Pamphlete. Aus der Schweiz. Nebst Briefen an Bodmern. 1781.

MLN Modern Language Notes

MLQ Modern Language Quarterly

MLR Modern Language Review

MsB Manuskript Bodmer bzw. -Breitinger

Nachw. Nachwort

NDB Neue Deutsche Biographie

Neue theatr. Werke Bodmer: Neue theatralische Werke. 1768.

N.F.

N.S.

Neue Folge

PMLA

Nuova Serie bzw. New Series

tion of America

Polit. Schausp. Bodmer: Politische Schauspiele. 1768-69.

Rez. Rezension

Schweiz. Schausp. Bodmer: Schweizerische Schauspiele. 1775.

Stäudlin

Briefe berühmter und edler Deutschen an Bodmer, hrsg. von Gotthold Friedrich Stäudlin. I794.

u.d.T. unter dem Titel

Vetter Bibliographie der Schriften J.J.Bodmers und der von ihm besorgten Ausgaben. In: Denkschrift (1900), S. 387-403.

Wolff Eugen Wolff: Briefwechsel Gottscheds mit Bod- 
mer und Breitinger. In: ZfdU ro (1887), S. 358 bis $38 \mathrm{r}$.

ZBZ

Zehnder

ZfdA

$\mathrm{ZfdPh}$.

ZfdU

Zürcher Tb.
Zentralbibliothek Zürich

Josephine Zehnder geb. Stadlin: Pestalozzi. Idee und Macht der menschlichen Entwicklung.

1875 .

Zeitschrift für deutsches Altertum

Zeitschrift für deutsche Philologie

Zeitschrift für den deutschen Unterricht

Zürcher Taschenbuch 